\title{
The Spanish Society for Applied Mathematics and ICIAM 2019 - Valencia
}

Rosa Donat (Universitat de València), President of the Sociedad Española de Matemàtica Aplicada

I would like to thank the editors of the EMS Newsletter, and especially Fernando Pestana da Costa, for the opportunity to make this presentation on the Spanish Society for Applied Mathematics (or Sociedad Española de Matemática Aplicada in Spanish, with acronym SeMA) in this very special year in which we will be the host society of ICIAM 2019, https://iciam2019.com, the ninth edition in the series of ICIAM congresses.

SeMA was created in 1991, following the success and continuity of the Spanish Congresses of Differential Equations and their Applications (CEDYA) that began in 1978. It was legally founded on February 25, 1993, but there are documents reporting a meeting in 1988 of a set of about twenty mathematicians, representing most of the Spanish research groups in Applied Mathematics that, led by Professor Antonio Valle, decided that the XI edition of CEDYA, to be held in Málaga

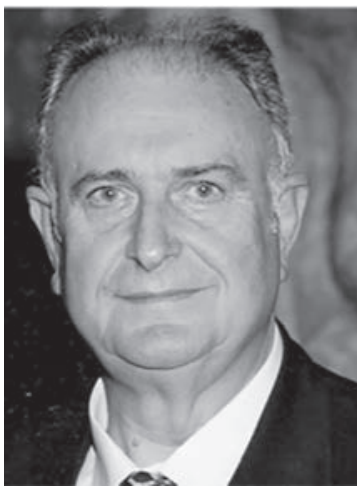
in 1989, would also be entitled "First Congress of Applied Mathematics" (I CMA). It was later, during the celebration of the XII CEDYA-II CMA at the Universidad de Oviedo in 1991, that more than a hundred mathematicians supported, by registering as members, the creation of SeMA as a society aiming to integrate researchers interested in applied mathematics living or working in Spain.

Professor Antonio Valle.

The mission envisioned for SeMA in its early years was to contribute in a coordinated manner to the development of mathematics in connection with its applications and the need to solve real-world problems in science and industry. The underlying motivation was the quantitative deep change that occurred in the preceding decade in the application of mathematical techniques and methodologies, due to the rapid development of scientific computing as a new emerging field.

During the last 25 years, SeMA has become a mature society, while contributing to the advancement of applied mathematics within the Spanish community. Nowadays the society has close to 500 individual members, some of them living or working outside of Spain, and around 50 institutional ones. Today the activity of SeMA is based upon the consideration that mathematical modelling, analysis, numerical simulation, and control techniques are essential tools to understand and solve many chal- lenging problems appearing in physics, chemistry, engineering, biomedical sciences, geosciences, economics, or finance, among others.

Its Executive Council is composed of 11 members: the president, a vice-president, the secretary general, the treasurer and 7 members elected for a period of three years and renewable for a second term. The president is elected for a period of two years, renewable for a second term. The Executive Council decides on all important matters concerning the society and meets at least once a year. The General Assembly of the society is open to all members of SeMA and meets once every year to approve the budget and discuss other matters.

SeMA holds cooperation agreements and reciprocity membership with many mathematical societies. In Spain, RSME - Royal Spanish Society of Mathematics, SCM - Catalan Society of Mathematics, SEIO - Spanish Society of Statistics and Operational Research, as well as the AMS and SIAM in the USA, SIMAI in Italy, SMAI in France, and GAMM in Germany. In addition, SeMA is a corporate member of several international mathematical organizations, such as the European Community on Computational Methods in Applied Sciences (ECCOMAS), the International Council for Industrial and Applied Mathematics (ICIAM), the International Mathematical Union (IMU, through the Spanish CEMAT, that is, the Comite Español de Matemáticas), and the Centre International de Mathématiques Pures et Appliquées (CIMPA). SeMA is a full member of the European Mathematical Society (EMS).

There are two special scientific events in our society. The CEDYA/CMA congresses, held every two years, have consolidated themselves as a focal meeting point for applied mathematicians in Spain. The week-long meetings include plenary talks by international experts, special minisymposia, contributed lectures, and poster sessions. The latest edition, http://www.cedya2017.org, was held in Cartagena in 2017. In alternate years, and in collaboration with the French SMAI, the society sponsors the Spanish-French Jacques-Louis Lions schools for graduate students. These schools are addressed to young researchers, specially pre- and post-doctoral applied mathematicians and engineers, as well as to industrial technicians interested in learning state-of-the-art numerical simulation techniques that may be useful in their field. The latest school was held in Las Palmas de Gran Canaria (http://ehf2018.iusiani.ulpgc.es).

In addition, the society has strived to consolidate its scientific publications. The scientific part of the 


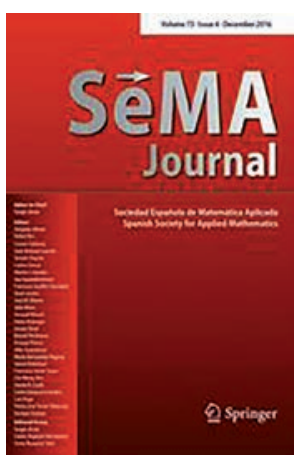

Boletín de la Sociedad Española de Matemática Aplicada, has become a peer-reviewed international scientific journal: the $\operatorname{SeMA}$ Journal, published by Springer since 2010. It contains articles and review papers written in English on high-level achievements in applied mathematics, covering theoretical as well as numerical results and also their practical applications. In order to promote the publication of high-quality scientific papers and reviews in applied mathematics, SeMA sponsors yearly the SeMA Journal Best Paper Award.

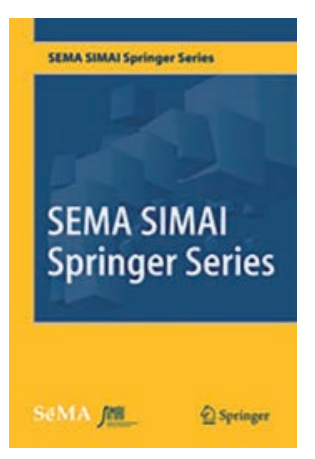

The cooperation SEMASIMAI Springer Series of advanced textbooks and research monographs deserves special mention. Launched in 2013, the series enjoys a very successful acceptance in the international applied mathematics community, and its impact is well above average. There are 18 volumes already published, and a few more are in production or waiting for final approval. Modelling and simulation are very much appreciated in our series, as we understand that they must be at the core of the mission of mathematics reaching out to society.

Our society also seeks to recognise the scientific contributions of young researchers by sponsoring the SeMA Prize to Young Researchers, established in 1998 and awarded annually to a young researcher under 34. This award was renamed the SeMA-Antonio Valle Prize to Young Researchers in 2013, in recognition of the efforts dedicated by Professor Antonio Valle, first president of SeMA, to the promotion of young researchers in the field of applied mathematics.

A special mention also goes to the collaboration between SeMA, SIMAI, SMAI, and SBMAC (the Brazilian society for applied mathematics), who presently fund the Lagrange Prize, created on the initiative of the first three societies and first awarded at the ICIAM 1999 congress. The prize was established to provide international recognition to individual mathematicians who have made an exceptional contribution to applied mathematics throughout their careers. The recipient of the 2019 Lagrange Prize is G. Papanicolaou 'for his brilliant use of mathematics to solve important problems in science and engineering; in particular, problems involving inhomogeneity, wave propagation, random media, diffusion, scattering, focusing, imaging, and finance' (http:// www.iciam.org/iciam-prizes-2019). The prize will be awarded at the opening ceremony of the ICIAM 2019 congress, on July 15, 2019.

As a full member of the International Council for Industrial and Applied Mathematics (ICIAM), SeMA was invited by ICIAM in 2011 to prepare a bid to host ICIAM 2019, the ninth ICIAM congress. After an inter- nal competition between several Spanish cities, Valencia was chosen as the venue for the conference in the SeMA bid, which had the support of the entire mathematical community in Spain as well as most of the ICIAM member societies in the Mediterranean area and many societies in Latin America and northern and equatorial Africa. Dr. Alfio Quarteroni, agreed to be the Chairman of the SPC (Scientific Committee Panel) and His Majesty King Felipe VI of Spain agreed to chair the Honour Committee of the conference, a fact of enormous importance for the social relevance of the event in the media. SeMA's bid was finally selected by the ICIAM Board in its 2013 meeting in Beijing, in fair competition with the bids presented by Amsterdam and Brazil. Since then, the society has been actively involved in the preparation of this large congress that will take place in Valencia, from July 15 to $19,2019$.

Organising ICIAM 2019 is certainly generating a qualitative, and hopefully also a quantitative, leap in the relations between mathematics and industry in our territory, and it has undoubtedly helped to raise the national assessment of applied mathematics, and the international liaison role of Spain between Europe, Latin America, and Africa.

There are many features that make an ICIAM conference a special event. The 27 plenary lectures do, of course, stand out: the speakers appointed by the SPC are researchers of global excellence with a variety of gender, thematic, and geographical origins. In addition, ICIAM2019-Valencia will have five additional talks corresponding to the five ICIAM awards (http://www.iciam. org/iciam-prizes-2019), as well as two special lectures: the Olga Taussky-Todd and the Outreach Public lecture. Information on all the distinguished speakers at ICIAM 2019 can be found at https://iciam2019.com/index.php/ scientific-program/.

As a new feature, ICIAM 2019 will hold a specific 'Industry Day', a mathematical and technology-transfer-oriented activity with a selection of speakers from industry, with a broad representation of the different sectors, whose target audience will be mainly the industrial attendees, but that is also addressed to the academic sector and open to the society in general.

ICIAM 2019 has, moreover, a special 'sub-venues' program. The Spanish cities of Bilbao, A Coruña, Málaga, Santiago de Compostela, Sevilla, and Zaragoza organise a dedicated program of satellite events, which reflect the commitment of the Spanish mathematical community with the success of the conference. The global Satellite Events Programme of ICIAM 2019 includes 30 events.

As of January 2019, ICIAM 2019 has already attracted more than 3500 talks. The call for minisymposia ended on December 5th (326 thematic minisymposia and 22 industrial minisymposia have been accepted). The contributed talks and posters submission periods end on January 21 and April 1, respectively.

SeMA and the organising committee of ICIAM 2019 have elaborated a Financial Aid Program for developing countries and young researchers (https:// 
iciam2019.org/index.php/infomation-for-delegates/ financial-support) that will provide funds for around 250 scholarships. Banco Santander deserves special recognition for sponsoring 150 grants for young students from all over the world. As usual, the ICIAM council helps to provide support for around 20 scholarships for developing countries. SeMA has contributed to the Financial Aid Program of ICIAM 2019 by implementing a crowdfunding program (P2B) addressed to Spanish institutions, which will provide funding for around 50 scholarships.

An ICIAM conference is a truly special event for the host society, but also for its immediate neighbourhood.
We invite all EMS members to join this thrilling journey across industrial and applied mathematics and to make ICIAM-2019-Valencia a great event of exceptional scientific quality.

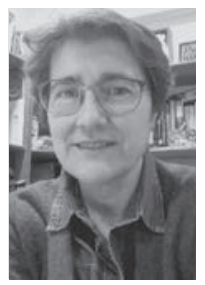

Rosa Donat is a professor at the Departament de Matemàtiques of the Universitat de València. Her research interests cover the numerical analysis of hyperbolic partial differential equations and the theory of nonlinear approximation, with applications to nonlinear subdivision schemes.

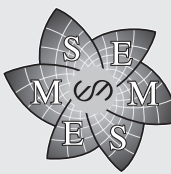

New journals published by the Guropean Mathematical Society
European Mathematical Society Publishing House Seminar for Applied Mathematics

ETH-Zentrum SEW A21, CH-8092 Zürich, Switzerland subscriptions@ems-ph.org / www.ems-ph.org

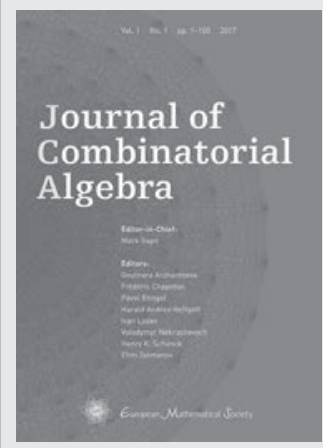

ISSN print $\quad 2415-6302$ ISSN online $2415-6310$ 2019. Vol. 3. 4 issues Approx. 400 pages. $17.0 \times 24.0 \mathrm{~cm}$ Price of subscription: 198 € online only / $238 €$ print+online

\author{
Editors-in-Chief: \\ Mark Sapir (Vanderbilt University, Nashville, USA)
}

\section{Editors:}

Goulnara Arzhantseva (University of Vienna, Austria), Frédéric Chapoton (CNRS and Universite de Strasbourg, France), Pavel Etingof (Massachussetts Institute of Technology, Cambridge, USA), Harald Andrés Helfgott (Georg-August Universität Göttingen, Germany and Centre National de la Recherche Scientifique (Paris VIIVII), France), Alexander Kleshchev (University of Oregon, Eugene, USA), Ivan Losev (Northeastern University, Boston, USA), Volodymyr Nekrashevych (Texas A\&M University, College Station, USA), Henry K. Schenck (Iowa State University, Ames, USA), Efim Zelmanov (University of California at San Diego, USA)

\section{Aims and Scope}

The Journal of Combinatorial Algebra is devoted to the publication of research articles of the highest level. Its domain is the rich and deep area of interplay between combinatorics and algebra. Its scope includes combinatorial aspects of group, semigroup and ring theory, representation theory, commutative algebra, algebraic geometry and dynamical systems. Exceptionally strong research papers from all parts of mathematics related to these fields are also welcome.

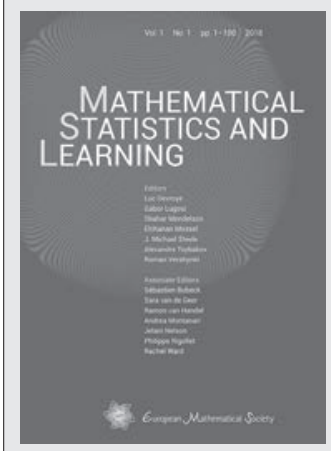

ISSN print $\quad 2520-2316$

ISSN online $2520-2324$

2019. Vol. 2.4 issues Approx. 400 pages. $17.0 \times 24.0 \mathrm{~cm}$ Price of subscription: $198 €$ online only / $238 €$ print+online

\section{Editors:}

Luc Devroye (McGill University, Montreal, Canada), Gabor Lugosi (UPF Barcelona, Spain), Shahar Mendelson (Sorbonne University, Paris, France and Australian National University, Canberra, Australia), Elchanan Mossel (MIT, Cambridge, USA), J. Michael Steele (University of Pennsylvania, Philadelphia, USA), Alexandre Tsybakov (CREST, Malakoff, France),

Roman Vershynin (University of California, Irvine, USA)

\section{Associate Editors:}

Sebastien Bubeck (Microsoft Research, Redmond, USA), Sara van de Geer (ETH Zurich, Switzerland), Ramon van Handel (Princeton University, USA), Andrea Montanari (Stanford University, USA), Boaz Nadler (Weizmann Institute of Science, Rehovot, Israel), Jelani Nelson (Harvard University, Cambridge, USA), Philippe Rigollet (MIT, Cambridge, USA), Rachel Ward (University of Texas, Austin, USA)

\section{Aims and Scope}

Mathematical Statistics and Learning will be devoted to the publication of original and highquality peer-reviewed research articles on mathematical aspects of statistics, including fields such as machine learning, theoretical computer science and signal processing or other areas involving significant statistical questions requiring cutting-edge mathematics. 